\title{
Mini-EUSO flight software and operations on ISS
}

\author{
F. Capel*, C. Fuglesang \\ KTH Royal Institute of Technology, Stockholm - Sweden \\ E-mail: capel@kth.se
}

\section{Casolino}

INFN - Sezione di Roma Tor Vergata, Italy

RIKEN - Japan

\section{Piotrowski}

RIKEN - Japan

\section{for the JEM-EUSO Collaboration}

\begin{abstract}
The Mini-EUSO instrument is designed by the JEM-EUSO collaboration to pave the way for space-based observations of Extreme Energy Cosmic Rays (EECRs). To be placed inside the International Space Station (ISS) in early 2018, it is a small UV (300 - $400 \mathrm{~nm}$ ) telescope which will observe the Earth's atmosphere with a spatial resolution of $6.11 \mathrm{~km}$. Mini-EUSO is capable of detecting a wide variety of UV events such as cosmic ray signals, transient luminous events and meteors with a minimum time resolution of $2.5 \mu \mathrm{s}$. It will also be possible to detect space debris during twilight periods. The flight software is fully automated and takes advantage of the frequent day/night cycles of the ISS orbit and ancillary instruments with which Mini-EUSO is equipped in order to optimise the mission's scientific output. The flight operations of MiniEUSO are presented including the data acquisition, storage and transfer, astronaut interaction and predicted instrument duty cycle.
\end{abstract}

35th International Cosmic Ray Conference ICRC 2017-

10-20 July, 2017

Bexco, Busan, Korea

${ }^{*}$ Speaker. 


\section{Introduction}

At the far end of the cosmic ray energy spectrum, with energies above $50 \mathrm{EeV}$, lie the EECRs. At such energies, the flux is as low as 1 particle $/ \mathrm{km}^{2} /$ century and the effective area that can be observed by a detector is a key feature. The JEM-EUSO Collaboration aims to detect the UV light produced by EECR-induced atmospheric showers from the vantage point of low Earth orbit, thereby largely increasing the effective detector volume $[1,2]$. In order to lay the groundwork for such unprecedented observations, the JEM-EUSO collaboration has successfully initiated several pathfinder experiments. Mini-EUSO forms the next step towards the observation of EECRs from space.

The main goal of Mini-EUSO is to measure the UV emissions from Earth orbit. These observations will provide interesting data for the scientific study of a variety of UV phenomena such as transient luminous events (TLEs), meteors, space debris, strange quark matter (SQM) and bioluminescence, as summarised in Figure 1. Firstly, Mini-EUSO will map the night-Earth, allowing the characterisation of the UV emission level, which is essential for the optimisation of the design of future EUSO instruments for EECR detection. TLEs are frequent UV-luminous lightning events which occur in the upper-atmosphere above typical electrical storms [3]. With its high time resolution of $2.5 \mu \mathrm{s}$, Mini-EUSO is capable of capturing these sub-ms events and to provide insight into their formation and development. Meteors emit in the UV as they burn up in the Earth's atmosphere and Mini-EUSO could be used in conjunction with ground based observations to study these phenomena in different wavelength channels. Sunlight reflected by small-scale space debris during twilight periods of observation could allow for the detection and high-resolution tracking of debris, as a prototype for future remediation systems. SQM is a hypothesised state of matter based on strange quarks which is a dark matter candidate [4]. Whilst it has never been detected, its interaction in the atmosphere would cause a UV trail, detectable by Mini-EUSO, as discussed in [5]. With its large exposure, Mini-EUSO will be capable of detecting SQM, or setting new upper limits on the flux of SQM for a null detection. Finally, whilst not optimised for the observation of large-scale bioluminescence, Mini-EUSO will be sensitive to such phenomena, allowing space-based observations to improve the understanding of these events.

Mini-EUSO is approved as a joint project by the Italian (ASI) and Russian (Roscosmos) space agencies and is included in the long-term program of space experiments on the Russian segment of the International Space Station (ISS) under the name "UV-Atmosphere". It is scheduled to be launched to the ISS, where it will be placed at a nadir-facing, UV-transparent window on the Russian Zvezda module. The integration of the instrument is currently at an advanced stage in order to be compliant with a launch opportunity in late 2017 to early 2018. For more details on the Mini-EUSO instrument and its diverse scientific goals, see [6].

\section{The Mini-EUSO instrument}

Mini-EUSO is based on one EUSO detection unit, referred to as the Photo Detector Module (PDM). The PDM consists of 36 multi-anode photomultiplier tubes (MAPMTs), each with 64 pixels, for a total of 2304 pixels. The MAPMTs are provided by Hamamatsu Photonics, model R11265-M64, and covered with a 2 mm BG3 UV filter with anti-reflective coating. The full Mini- 


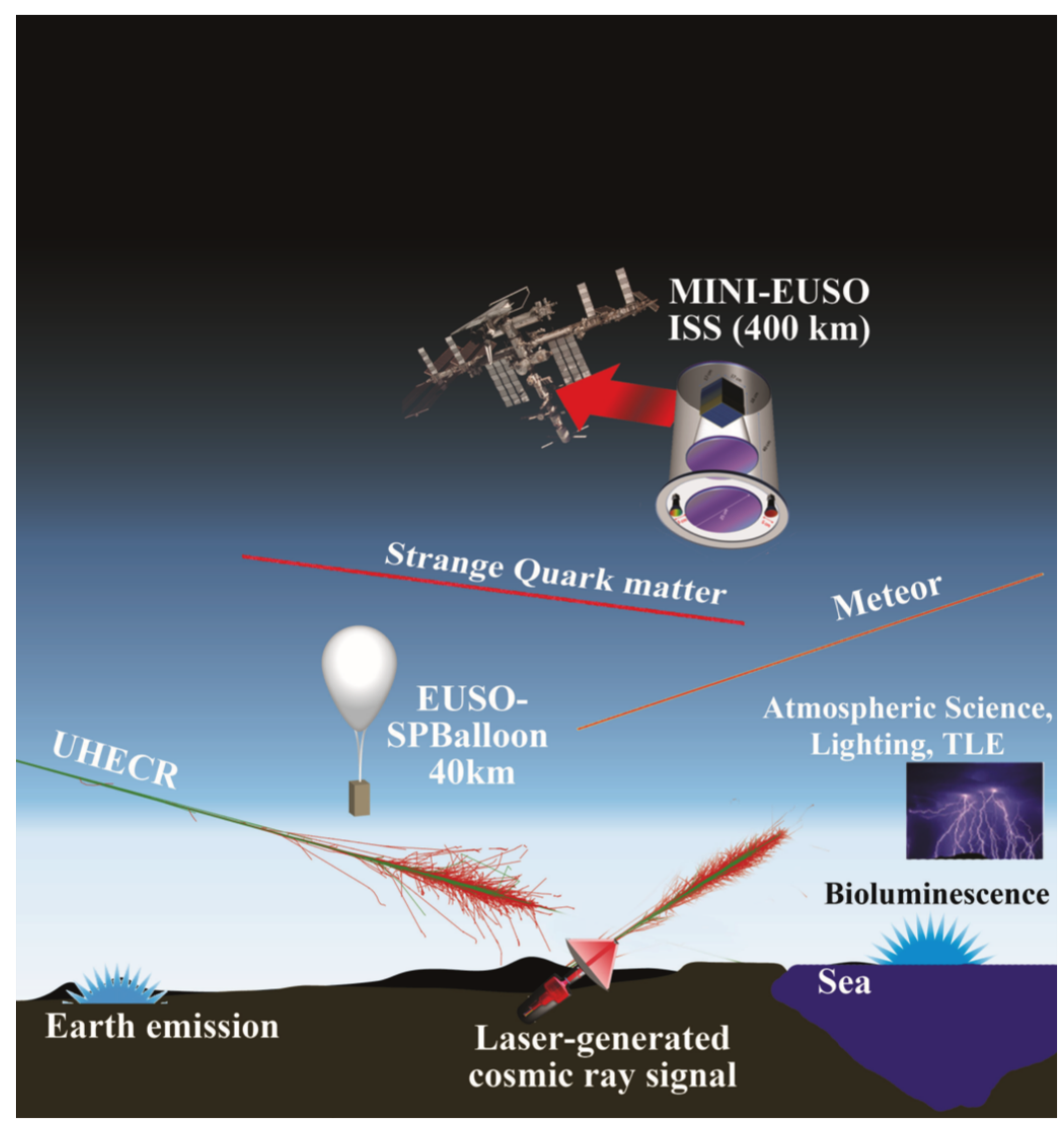

Figure 1: The Mini-EUSO mission summarised in one diagram. From the ISS, Mini-EUSO will observe a variety of interesting phenomena in the UV range, in addition to creating a high resolution UV map of the Earth.

EUSO telescope is made up of 3 main systems, the optical system, the PDM and the data acquisition system. The optical system of 2 Fresnel lenses is used to focus light onto the PDM in order to achieve a large field of view $\left(44^{\circ}\right)$ with a relatively light and compact design $(0.8 \mathrm{~kg}$ per lens, and a focal length of $30 \mathrm{~cm}$ ), well-suited for space application. The PDM detects UV photons and is read out by the data acquisition system with a sampling rate of $2.5 \mu \mathrm{s}$ and a spatial resolution of $6.11 \mathrm{~km}$.

In addition to the main detector, Mini-EUSO contains two ancillary cameras for complementary measurements in the near infrared (NIR from 1500 to $1600 \mathrm{~nm}$ ) and visible (VIS from 400 to $780 \mathrm{~nm}$ ) range. The main task of the cameras is to provide multi-wavelength monitoring in order to better understand the UV luminosity measurements. These cameras are placed outside the lenses at the front of the instrument and acquire data independently of the PDM. At the edges of focal surface, an array of silicon photomultipliers (SiPMs) is placed in order to be tested as an alternative detection technology for potential use in future EUSO missions. The complete Mini-EUSO instrument is contained in a box with a connection to ISS for power/grounding and the interface to the UV-transparent window, from which Mini-EUSO will observe in a fixed position on-board the ISS, facing the nadir direction. Figure 2 shows the layout of the main systems. 
The array of MAPMTs in the Mini-EUSO PDM is powered by a Cockroft-Walton high voltage power supply (HVPS), allowing for a low power solution to providing the high voltage needed by the PMTs. In order to protect the MAPMTs from potentially damaging high current levels, the HVPS has a fast $(<3 \mu \mathrm{s})$ built-in switch which drastically reduces the gain of the MAPMTs when the anode current surpasses a threshold value. The photon flux of the phenomena which will be observed by Mini-EUSO varies on the order of $10^{6}$, from background levels of 1 count/pixel/GTU $(1 \mathrm{GTU}=2.5 \mu \mathrm{s})$, to bright TLEs and meteors. The HVPS handles this large range via the implementation of a second switch, controlled by the Zynq board. This second switch works by reducing the gain in a controlled way, as the incident photon flux increases in order to cover the full dynamic range of $10^{6}$.

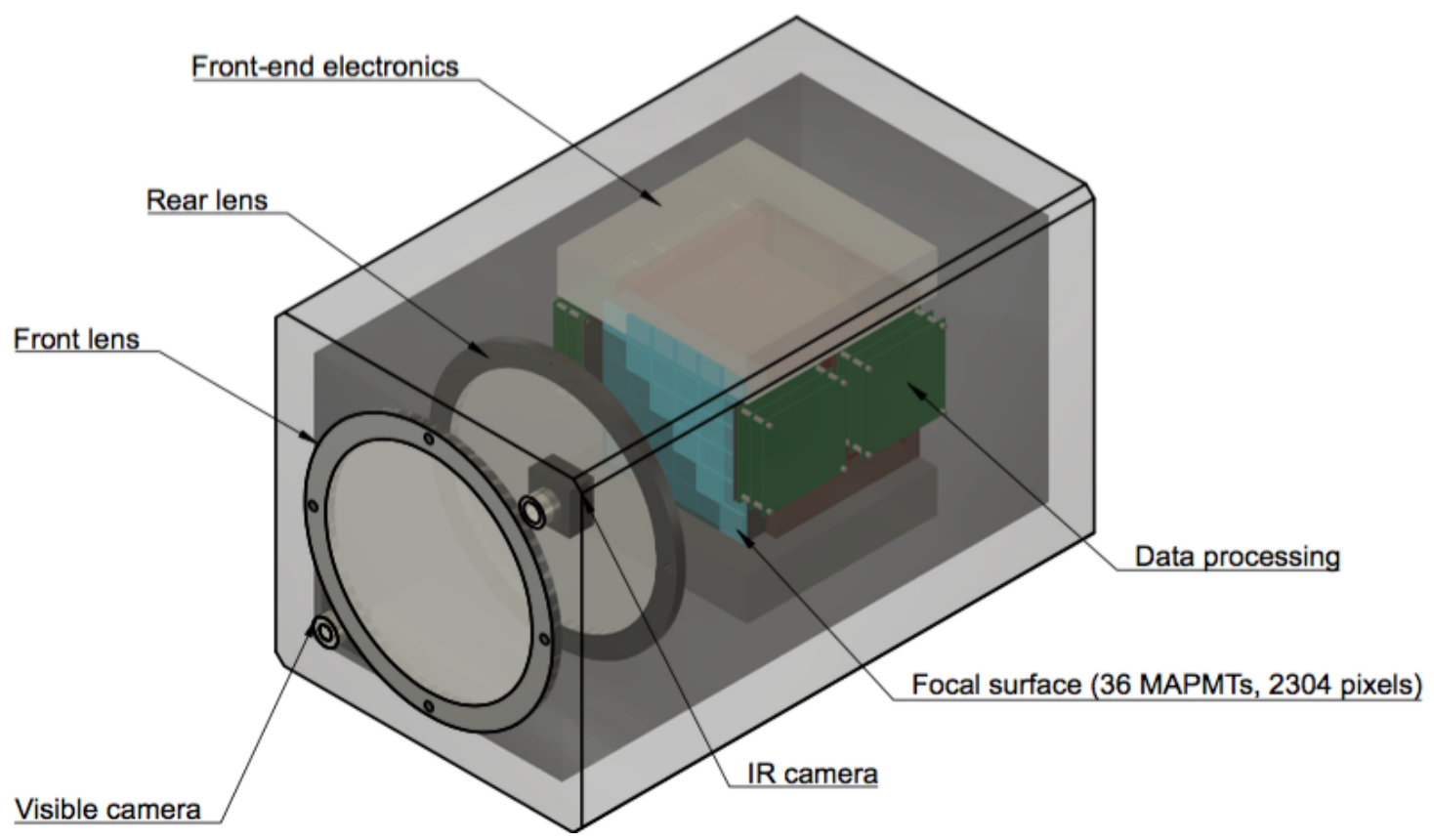

Figure 2: Mini-EUSO conceptual design. The optical system with two double sided Fresnel lenses $(25 \mathrm{~cm}$ diameter) focuses the UV light on to a focal surface consisting of a single PDM, made up of 36 MAPMTs and 2304 pixels. Ancillary detectors are the VIS and NIR cameras. The instrument dimensions are $37 \times 37$ $\times 62 \mathrm{~cm}^{3}$.

\section{The flight software}

Mini-EUSO will be situated inside the Russian Zvezda module at a nadir-facing, UV-transparent window. For simplicity, the only connection to the ISS will be be for power $(30 \mathrm{~W})$ and grounding, with no continuous telemetry capability. In order to be able to achieve its various scientific goals, the Mini-EUSO instrument must function autonomously during its flight, with minimal astronaut interaction. The ISS flies in low Earth orbit at an altitude of $\sim 400 \mathrm{~km}$ with an orbital speed of $\sim 8 \mathrm{~km} \mathrm{~s}^{-1}$. From the vantage point of the Russian Zvezda module, Mini-EUSO will have a roughly uniform coverage of the entire Earth's surface excluding the polar regions. The orbital pe- 
riod of 93 min means that the ISS passes from local day to local night roughly every $46.5 \mathrm{~min}$. The main Mini-EUSO data acquisition can only take place during the local night-time, in order for the MAPMTs to operate without damage in single-photon counting mode. In this way, the instrument must be capable of switching between operational modes and making efficient use of the frequent day/night cycle.

The data acquisition chain consists of the front-end electronics, the PDM-DP (PDM data processing) based on a Xilinx Zynq XC7Z030 [8] system, and a PCIe/104 CPU. Incoming photon pulses are pre-amplified and digitised by the SPACIROC3 ASICs [9] an integrated over a period of $2.5 \mu \mathrm{s}$, referred to as a GTU. The signal then passes through a multi-level trigger system [10] which is designed to capture events of interest on different time scales and is implemented in the hardware of the Zynq FPGA, before being passed to the CPU. The data acquisition system is summarised in Figure 3. The flight software is implemented in $\mathrm{C} / \mathrm{C}++$ on the $\mathrm{CPU}$, and performs the control of the instrument sub-systems as well as the data management and storage, housekeeping, switching between operational modes and collecting data from the NIR and VIS cameras. Data is stored on board in SSDs which are periodically returned to Earth from the ISS, as it is not possible to telemeter the expected amount of $\sim 2 \mathrm{~TB} /$ month. In order to monitor the status of the Mini-EUSO instrument, smaller compressed data samples $(\sim 200 \mathrm{MB})$ containing diagnostic information on the instrument status will be telemetered at regular intervals by the astronauts on board the ISS.

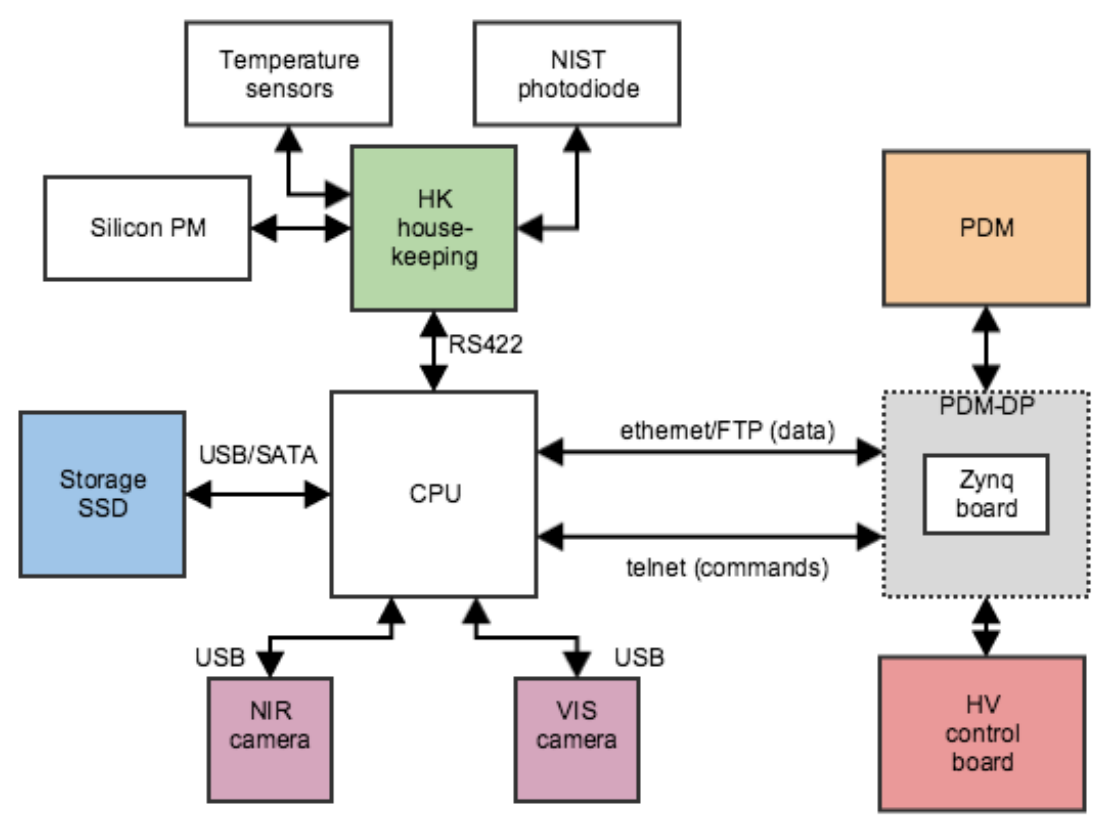

Figure 3: The data acquisition system of Mini-EUSO with the main interfaces shown. Incoming data from the PDM ASICs is triggered in the Zynq board and then passed to the CPU via an ethernet link. The CPU combines this data with that of the housekeeping system and the ancillary instruments: NIR, VIS, photodiode, and SiPMs. Data is then moved to on-board SSDs for storage. 


\subsection{Night-time observations}

Upon switching on, Mini-EUSO makes use of 4 calibrated photodiodes with set thresholds in order to determine its operational mode. These are placed at the level of the focal surface, adjacent to the array of MAPMTs. If the UV flux is below the maximum permitted by the HV switching system of the MAPMTs $\left(10^{6}\right.$ photons/pixel/GTU), Mini-EUSO will function in the night-time operational mode. In this mode, data gathering is driven by the multi-level trigger algorithm, which outputs data on three timescales every $5.24 \mathrm{~s}$ : L1 data with a resolution of $2.5 \mu \mathrm{s}$, L2 data with a resolution of $320 \mu$ s and L3 data with a resolution of $40.96 \mathrm{~ms}$. L1 is optimised for the detection of EECRs and EECR-like signals generated by ground based laser systems for the verification and in-flight calibration of the instrument. L2 is designed to capture TLEs and lightning in the upperatmosphere to provide high resolution measurements of the development of these phenomena. The L3 readout will provide a continuous "movie" of the UV emissions of the Earth for mapping of the background to EECR signals with unprecedented detail. The L3 data can also be used in conjunction with offline trigger algorithms to study meteors, nuclearites, space debris and other slower phenomena.

The NIR and VIS cameras will operate with a trigger passed from the Mini-EUSO PDM in order to provide multi-wavelength measurements of slower atmospheric events, such as meteors and space debris. When not triggered, the cameras will operate continuously to provide complementary measurements on the atmospheric status at the time of measurement, matching the third level of data from the Mini-EUSO trigger. In addition to this, housekeeping data from the thermistors, the silicon photomultiplier array and the photodiodes will be read out via analog channels every $5.24 \mathrm{~s}$. This data is organised, packaged and stored throughout the night-time operations which have a typical duration of $42.5 \mathrm{~min}$. The data output rate in this mode is estimated to be $\sim 2 \mathrm{~TB} / \mathrm{month}$ (of which $\sim 1.3 \mathrm{~TB} /$ month is the main data from the PDM), assuming a $25 \%$ duty cycle.

\subsection{Day-time operations}

Once the UV flux measured by the photodiodes surpasses the threshold for the safe operation of the MAPMTs, the instrument switches to day-time operations; the HV power to the MAPMTs is ramped down and the triggered data acquisition is stopped. During the local day-time, the CPU processing power is focused on the compression and filtering of data from the previous night-time run, in order to produce a so-called "quick-look" sample to be telemetered to ground during the flight. It is expected to have a small data budget of $\sim 200 \mathrm{MB} /$ month for such purposes. This budget would initially be allotted to the transfer of diagnostic and housekeeping information on the various subsystems at early stages in the mission, in order to verify the operation of the instrument and fine-tune its configuration. At later times in the mission, it is planned to send packets containing strong candidate events for the L1 and L2 output data levels, filtered using dedicated algorithms running during the day-time operations. In addition, the ancillary cameras will continue to operate at a reduced sample rate during the day with a continuous readout for atmospheric monitoring, accompanied by housekeeping data read out at the same frequency of $\sim 0.5 \mathrm{~Hz}$. This reduced data sample will be acquired and stored in the same manner as the acquisition of the night-time operations. 


\subsection{Further considerations}

During each day-night transition, there is a period of a few minutes during which the ISS and the Mini-EUSO instrument are in darkness but the lower atmosphere is still lit by the sun. At this point in time, it would be possible for Mini-EUSO to detect the UV light reflected by smallscale space debris below the ISS orbit, as detailed in [11]. This concept is still being studied and simulations are currently underway in order to define the ideal operational mode of the instrument at this time.

Mini-EUSO will have a limited amount of astronaut interaction in order to ensure the correct positioning and operation of the instrument during its mission lifetime. Astronaut time will be scheduled for the connection and switching on of the instrument, in addition to regular checks of the status of the replaceable SSDs used for the bulk data storage. A 90\% full SSD will be indicated by the presence of a corresponding red LED in the instrument status panel. Additionally, a removable USB drive will be present for the storage of quick-look data and the configuration file. As well as downlinking the quick-look data it will be possible to upload a configuration file for the editing of important parameters in the automated software. The software is designed to be as configurable as possible, allowing adaptation of the instrument operations to off-nominal situations. Configurable parameters include the thresholds and integration periods used in the trigger logic, the pixel mask for the MAPMTs, the photodiode threshold for switching operational mode and the type of quick-look data to be stored on the removable USB.

\section{Implementation and testing}

The flight software for the Mini-EUSO engineering model has been developed and tested. The main automated data acquisition has been verified along with the integration of ancillary instruments and the house-keeping system. The testing of the software currently proceeds in parallel with the development of the flight model, including end-to-end tests of the instrument data flow and storage, making use of both simulated data and flight data of the recent EUSO-SPB instrument (a similar prototype to the Mini-EUSO instrument flown on a NASA Super-Pressure Balloon in May [12]), passed directly to the front-end electronics.

\section{Conclusion}

The Mini-EUSO instrument will fly on the ISS with an automated software designed to take advantage of the frequent day/night cycles of the ISS orbit and ancillary instruments with which Mini-EUSO is equipped in order to optimise the mission's scientific output. This software is currently under development, in parallel with the flight model construction, and the basic functionality of the software has been validated using the engineering model of the instrument. The final flight software will be completed and tested by September 2017, in order to be compliant with a launch opportunity in late 2017 or early 2018. 


\section{Acknowledgments}

This work was partially supported by the Italian Ministry of Foreign Affairs and International Cooperation, Italian Space Agency (ASI) contract 2016-1-U.0, State Space Corporation ROSCOSMOS, the Russian Foundation for Basic Research, grant \#16-29-13065, and the Olle Engkvist Byggmästare Foundation. We dedicate this proceeding to Yoshiya Kawasaki and Jacek Karczmarczyk, who passed away in 2016.

\section{References}

[1] The JEM-EUSO Collaboration, The JEM-EUSO instrument, Experimental Astronomy 40(1) (2015) $19-44$

[2] A. V. Olinto, E. Parizot, M. Bertaina, and G. Medina-Tanco, JEM-EUSO Science In: Proceedings of the 34th International Cosmic Ray Conference (2015)

[3] Pasko, V.P., Yair, Y. \& Kuo, C.-L., Lightning Related Transient Luminous Events at High Altitude in the Earth's Atmosphere: Phenomenology, Mechanisms and Effects, Space Science Reviews 168 (2011) 475-516

[4] De Rujula, A. \& Glashow, S.L., Nuclearites: A Novel Form of Cosmic Radiation, Nature 312(5996) (1984) 734-737

[5] Adams, J.H., Jr et al., JEM-EUSO: Meteor and nuclearite observations, Experimental Astronomy 40(1) (2014) 253-279

[6] F. Capel et al. (JEM-EUSO Coll.), Mini-EUSO: A high resolution detector for the study of terrestrial and cosmic UV emission from the International Space Station submitted to Advances in Space Research (2017)

[7] Bacholle, S. et al., A Cockcroft-Walton High-Voltage Power Supply for the EUSO Instruments, In: Proceedings of the 34th International Cosmic Ray Conference (2015)

[8] Xilinx. Zynq-7000 All Programmable SoC, URL: https://www.xilinx. com/products/silicon-devices/soc/zynq-7000.html (2016)

[9] Blin-Bondil, S, Barrillon, P, and Dagoret-Campagne, S. SPACIROC3: A Front-End Readout ASIC for JEM-EUSO cosmic ray observatory In Proceedings of the 3rd International Conference on Technology and Instrumentation in Particle Physics (TIPP), Amsterdam (2014)

[10] A. Belov, F. Capel, F. Fausti et al. (JEM-EUSO Coll.), The integration and testing of the Mini-EUSO multi-level trigger system, submitted to Advances in Space Research (2017)

[11] T. Ebisuzaki et al., Demonstration designs for the remediation of space debris from the International Space Station, Acta Astronautica 112 (2015) 102-113

[12] L. Wiencke, J. Adams, and A. Olinto, The EUSO-SPB Mission, In Bulletin of the American Physical Society (2016) 